\title{
Topological scattering resonances at ultralow frequencies
}

\author{
S. Ali Hassani Gangaraj, ${ }^{1, *}$ Constantinos Valagiannopoulos $\odot,{ }^{2, \dagger}$ and Francesco Monticone $\odot^{1, \ddagger}$ \\ ${ }^{1}$ School of Electrical and Computer Engineering, Cornell University, Ithaca, New York 14853, USA \\ ${ }^{2}$ Department of Physics, School of Sciences and Humanities, Nazarbayev University, Astana KZ-010000, Kazakhstan
}

(Received 21 November 2019; accepted 9 April 2020; published 15 May 2020)

\begin{abstract}
At frequencies much lower than the plasma frequency, individual subwavelength plasmonic scatterers are typically far from any scattering resonance, with the exception of some extreme geometries. Contrary to this conventional behavior, in this paper, we theoretically demonstrate that the application of a weak magnetic bias to a portion of the plasmonic scatterer leads to the emergence of scattering resonances that exist, in principle, for an arbitrarily low frequency. We show that this class of plasmonic resonances originates from ultralow-frequency unidirectional and topological surface modes that emerge at internal interfaces. These topological properties endow the scattering resonances with high robustness against geometrical modifications. Our findings may open new uncharted directions towards the design of robust, shape-independent, subwavelength resonant structures with extreme scattering response.
\end{abstract}

DOI: 10.1103/PhysRevResearch.2.023180

\section{INTRODUCTION}

Scattering resonances in individual subwavelength objects are of fundamental importance to enhance the interaction of electromagnetic fields with matter, and to realize artificial metastructures with exotic effective properties. At optical frequencies, particular attention has been devoted to metallic scatterers, which resonate at frequencies close to the plasma frequency $\omega_{p}$ of their constituent materials. These resonances are, in principle, independent of the object size, which can therefore be deeply subwavelength [1,2]. However, at frequencies much lower than $\omega_{p}$, an individual subwavelength plasmonic element, e.g., a sphere or a wire/cylinder, is typically far from any scattering resonances, with a couple of relevant exceptions.

There are indeed two conventional strategies for making an individual plasmonic element resonate at extremely low frequencies; however, both involve a singular geometry with vanishing thickness. For example, spheroidal or ellipsoidal particles exhibit resonances for values much lower than the plasma frequency. The resonant frequency scales as $\omega_{p} \sqrt{L}$, where $L$ is a shape parameter called the depolarization factor [3]. For the case of a prolate spheroid, for instance, the depolarization factor can reach zero asymptotically, corresponding to an extremely sharp and thin needle of vanishing thickness [3]. Another strategy to lower the plasma frequency is to consider a core-shell sphere, with a plasmonic shell and a dielectric core. A particle of this type exhibits two resonances, a symmetric (bonding) and an antisymmetric (antibonding)

\footnotetext{
*ali.gangaraj@gmail.com

${ }^{\dagger}$ konstantinos.valagiannopoulos@nu.edu.kz

‡francesco.monticone@cornell.edu
}

Published by the American Physical Society under the terms of the Creative Commons Attribution 4.0 International license. Further distribution of this work must maintain attribution to the author(s) and the published article's title, journal citation, and DOI. resonance, due to the coupling between surface plasmon modes on the two spherical interfaces. In particular, the symmetric resonance shifts towards lower frequencies as the thickness of the shell approaches zero, i.e., for extremely thin shells [4]. Interestingly, the same shifting phenomenon is valid for any core-shell geometry with a plasmonic shell of vanishing thickness. Thus, while it is possible to arbitrarily lower the resonant frequency of individual plasmonic elements, these strategies require a geometry with vanishing features, that is, infinitesimally thin needles or infinitesimally thin shells. In this paper, we propose a very different approach to realize a scattering resonance at extremely low frequencies.

Metals and plasmas are opaque below $\omega_{p}$ : the allowed bulk modes are evanescent waves, whereas surface modes (surface plasmon polaritons), at an interface with, e.g., free space, exist at arbitrarily low frequencies, but they are highly confined only at frequencies close to $\omega_{p} / \sqrt{2}$. This behavior is significantly modified if an external static magnetic field is introduced. Indeed, it has been known for decades that the application of a magnetic bias to a plasma may open new propagation channels at ultralow frequencies [5]. This is especially important in atmospheric physics, where it takes the name of "whistler effect": the Earth's magnetic field opens propagation channels (whistler modes) in the ionosphere plasma at audio frequencies. Without the magnetic bias, the plasma would be completely opaque at these frequencies (relative permittivity $\epsilon \approx-10^{5}$ ). Inspired by the opportunities offered by applying a suitable magnetic bias to a plasmalike material, in this paper we theoretically demonstrate the possibility of realizing scattering resonances in individual subwavelength plasmonic objects at frequencies arbitrarily lower than the plasma frequency.

\section{ULTRALOW-FREQUENCY SCATTERING RESONANCES}

Consider an infinitely long plasmonic cylinder composed of a metallic material following a standard lossless Drude model, with relative permittivity $\epsilon=1-\omega_{p}^{2} / \omega^{2}$. The 
structure is illuminated by a transverse-magnetic (TM) plane wave with magnetic field parallel to the cylinder axis. In the quasistatic regime, namely, if the free-space wavelength $\lambda$ at the frequency of interest $\omega$ is much larger than the diameter $2 b$ of the cylinder, the first multipolar scattering contribution that can exhibit a resonance is the electric dipolar one [6]. By applying the quasistatic approximation, and neglecting radiation loss for simplicity, the corresponding scattering coefficient can be written as [6]

$$
S_{ \pm 1} \approx \mp \pi^{3}\left(\frac{b}{\lambda_{p}}\right)^{2} \frac{\left(\omega / \omega_{p}\right)^{2}}{1-2\left(\omega / \omega_{p}\right)^{2}}
$$

where $\lambda_{p}$ is the free-space wavelength at $\omega_{p}$. The $m= \pm 1$ subscript refers to dipolar terms of equal and opposite angular momentum (related to the azimuthal angular dependence $e^{i m \phi}$ of the scattered wave, where $\phi$ is the cylindrical coordinate angle measured from the $+x$ axis). Written in this way, Eq. (1) reveals that the dipolar scattering response is determined by two independent ratios: the geometrical ratio $b / \lambda_{p}$ and the frequency ratio $\omega / \omega_{p}$. This formula expresses the well-known fact that, in the lossless quasistatic limit, a subwavelength plasmonic cylinder resonates at a frequency $\omega=\omega_{p} / \sqrt{2}$, i.e., the localized surface-plasmon resonance, regardless of the object's size. Indeed, the ratio $b / \lambda_{p}$ can be arbitrarily small at resonance, meaning that, for example, a nanometer-scale plasmonic object can resonate under wavelengths in the micrometer range. Instead, the resonance frequency is only moderately smaller than $\omega_{p}$, implying that a subwavelength plasmonic cylinder with plasma frequency at optical frequencies cannot resonate at microwaves, as expected. Although this is a widely accepted fact, here we show that a simple approach allows lowering the frequency of this scattering resonance by orders of magnitude (in principle, for $\omega / \omega_{p} \rightarrow 0$ ).

Incidentally, we note that one of the first breakthroughs of the field of metamaterials was indeed the possibility of obtaining ultralow-frequency plasmonic effects, drastically lowering the effective plasma frequency of the material by structuring it in the form of wire meshes or analogous structures [7,8]. This is completely different from our approach, in which the plasma frequency remains unchanged, but we obtain plasmonic resonances extremely far from it.

Equation (1) also shows that the scattering coefficients for dipolar terms of equal and opposite angular momentum, $m= \pm 1$, differ only by an opposite sign, and resonate at exactly the same frequency. Hence, this scattering resonance is doubly degenerate. Such a degeneracy ultimately originates from the fact that the surface plasmon polariton supported by the surface of the scatterer exhibits perfectly symmetric propagation properties clockwise or counterclockwise, which is a direct consequence of the time-reversal symmetry of this electromagnetic configuration (equivalent to Lorentz reciprocity in the lossless case). It is therefore natural to expect that such a scattering resonance degeneracy can be lifted by breaking time-reversal symmetry and reciprocity. The simplest way to do so is by biasing the system with a static magnetic field along the cylinder axis (or, alternatively, by spinning the cylinder). The application of the static magnetic bias along the $z$ axis makes the plasmonic material anisotropic and gyrotropic, characterized by a nonsymmetric tensor of the form $\underline{\boldsymbol{\epsilon}}_{1}=\left(\epsilon_{t} \mathbf{I}_{t}+\epsilon_{a} \hat{\mathbf{z}} \hat{\mathbf{z}}+i \epsilon_{g} \hat{\mathbf{z}} \times \mathbf{I}\right)$, where $\left(\mathbf{I}_{t}=\mathbf{I}-\hat{\mathbf{z}} \hat{\mathbf{z}}\right)$ and $\mathbf{I}$ is a $3 \times 3$ identity matrix. The elements of this tensor follow the standard dispersion model of a magnetized Drude plasma [5] (a time-harmonic dependence $e^{+i \omega t}$ is assumed for all field quantities and suppressed). Plane-wave scattering from an infinite gyrotropic cylinder can be solved exactly using a generalized Mie theory $[6,9,10]$. Since we are interested in deeply subwavelength scatterers, we consider $b / \lambda \ll 1$ and perform a quasistatic analysis (see Appendix A for further details on the validity of this analysis). Under this assumption, the dominant scattering coefficients are the dipolar terms $m=$ \pm 1 , corresponding again to two circularly polarized dipoles with opposite handedness; however, their degeneracy is now lifted. The dipolar scattering coefficients $S_{ \pm 1}$, in the longwavelength regime, are given by [10]

$$
S_{ \pm 1} \approx \mp \pi^{3}\left(\frac{b}{\lambda_{p}}\right)^{2} \frac{\left(\omega / \omega_{p}\right)^{2}}{1-2\left(\omega / \omega_{p}\right)^{2} \mp 2 \frac{\omega_{c}}{\omega}\left(\omega / \omega_{p}\right)^{2}} .
$$

The bias-induced resonance splitting (Zeeman effect) is determined by the additional term in the denominator, which depends linearly on the cyclotron frequency $\omega_{c}$ [10]. Clearly, while this effect provides a mechanism to change and tune the plasmonic resonance, only large bias intensities allow moving the resonance to frequencies much lower than $\omega_{p}$.

Another popular strategy to tune plasmonic resonances is to consider core-shell geometries, which introduce new interfaces supporting surface plasmon-polaritons and allow realizing multiple resonances [11]. Inspired by this strategy, we analyzed the scattering response of a column of plasma that is only partially magnetized, realizing a cylindrical core-shell structure, as schematically shown in the inset of Fig. 1(a). The gyrotropic (magnetized) core, with radius $a$, is surrounded by an isotropic nonmagnetized layer with the same plasma frequency $\omega_{p}$ and outer radius $b$. In Appendix B we discuss potential materials for the practical implementation of this scatterer, and we comment on the feasibility of this bias configuration. At this point, we should also stress that, while the assumption of an identical plasma frequency for the core and shell allows greatly simplifying the analysis, it is not a requirement to observe the effects discussed below, which remain qualitatively similar even if the shell material has a different plasma frequency (as long as it remains an opaque medium at the frequency of interest).

The dipolar scattering coefficients $S_{ \pm 1}$ of the considered core-shell structure, in the long-wavelength regime, are now given by a more complicated expression provided in Appendix A. By comparing this expression with Eq. (2), we note that the scattering coefficient admits an additional pole, indicating the presence of an additional dipolar scattering resonance. Rather strikingly, this resonance exists at arbitrarily low frequencies. Indeed, under the assumption $\omega / \omega_{p} \ll 1$, we can write this new resonance condition in closed form as

$$
\frac{\omega}{\omega_{p}}=\frac{\left|\omega_{c}\right|}{2 \omega_{p}}\left[1-\left(\frac{a}{b}\right)^{2}\right],
$$

which corresponds to the $S_{-1}$ coefficient if the bias is along the $+z$-axis. The resonance condition for the scattering coefficient $S_{+1}$ is located symmetrically at a negative frequency. Equation (3) reveals that the scattering resonance can be 


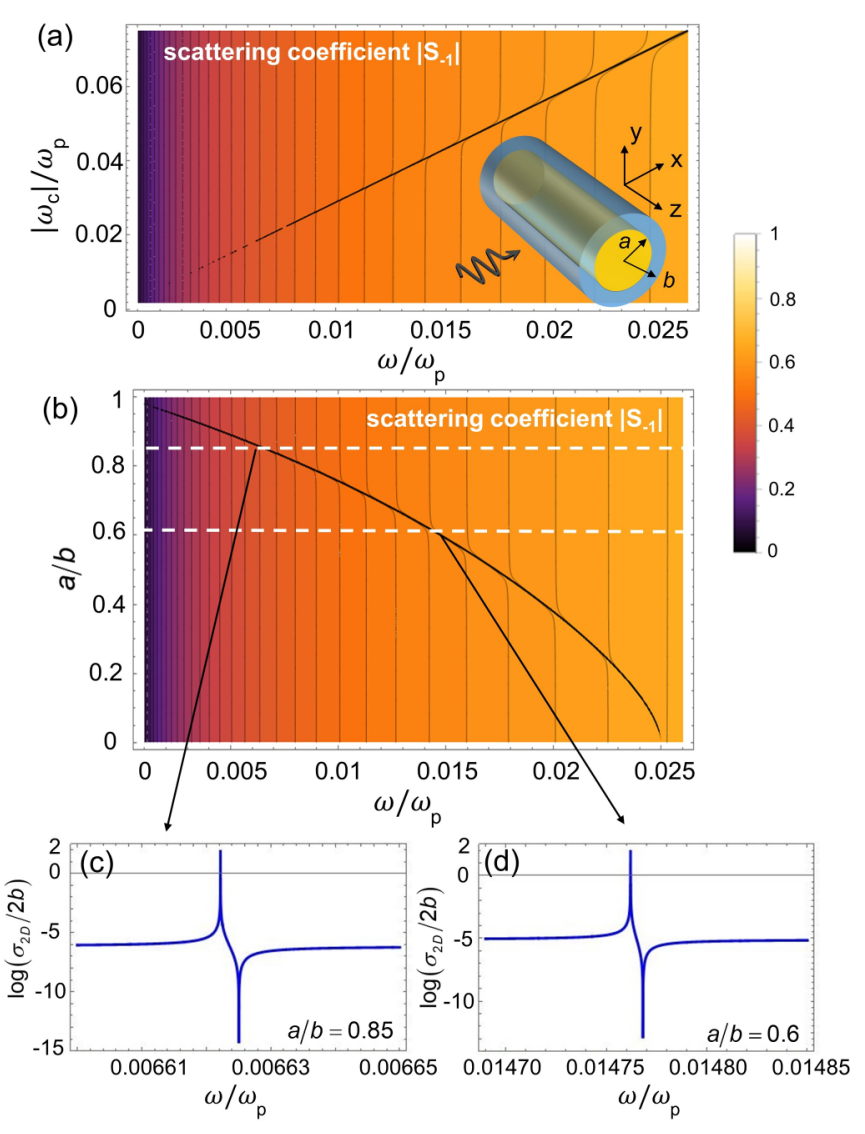

FIG. 1. Ultralow-frequency scattering resonance in a partially biased plasmonic cylinder. (a) Dipolar scattering coefficient, $\left|S_{-1}\right|$, obtained by exact Mie-theory calculations, for the core-shell cylinder in the inset, as a function of normalized frequency, $\omega / \omega_{p}$, and cyclotron frequency, $\omega_{c} / \omega_{p}$, for an aspect ratio $a / b=0.5$. (b) Same as panel (a), but varying frequency, $\omega / \omega_{p}$, and aspect ratio, $a / b$, with a fixed cyclotron frequency $\omega_{c} / \omega_{p}=0.05$. Core and shell have the same plasma frequency, $\omega_{p}$, but only the core is biased (along the $+z$ axis). The outer radius is fixed to $b / \lambda_{p}=0.2$. (c), (d) Scattering efficiency, $\sigma_{2 D} /(2 b)$, for (c) $a / b=0.85$ and (d) $a / b=0.6$.

lowered arbitrarily, asymptotically approaching zero frequency, by either increasing the aspect ratio $a / b \rightarrow 1$ (decreasing the shell thickness) or by decreasing the cyclotron frequency $\omega_{c} \rightarrow 0$. We also note that, as the resonance approaches zero frequency, for $\omega_{c} \rightarrow 0$ or $a \rightarrow b$, the scattering pole (resonance) is canceled by a scattering zero as $\omega \rightarrow 0$, and the scattering coefficient vanishes in this limit, i.e., $S_{-1} \rightarrow 0$. At exactly zero frequency the scattering coefficient is zero for any choice of parameters.

As mentioned in the Introduction, such a resonance shift towards zero frequency is similar to the behavior of a reciprocal core-shell scatterer, with nonbiased plasmonic shell and dielectric core, as the thickness of the shell vanishes. There are, however, some relevant differences. Through a quasistatic analysis of a conventional core-shell cylindrical scatterer, a resonance condition similar to Eq. (3) can be found in the limit of very small frequency:

$$
\frac{\omega}{\omega_{p}}=\frac{\sqrt{1-\left(\frac{a}{b}\right)^{2}}}{\sqrt{3+\left(\frac{a}{b}\right)^{2}(\epsilon-1)+\epsilon}},
$$

where the shell follows a lossless Drude model with plasma frequency $\omega_{p}$ and the core has permittivity $\epsilon$. A comparison of Eqs. (3) and (4) reveals that, in both cases, the scattering resonance indeed approaches zero frequency if $a \rightarrow b$, but such a resonance shift is faster for the proposed gyrotropic scatterer. Most importantly, Eq. (3) also shows that, even if the shell has nonzero thickness, the resonance can be lowered arbitrarily by lowering the cyclotron frequency. This is very different from the behavior of conventional plasmonic scatterers, for which a scattering resonance can approach zero frequency only if the geometry has some vanishing features, as in the case of infinitesimally thin plasmonic needles or infinitesimally thin plasmonic shells.

To further confirm this behavior, we show in Fig. 1(a) the scattering coefficient magnitude $\left|S_{-1}\right|$ for the considered gyrotropic core-shell cylinder, as a function of frequency $\omega / \omega_{p}$ and cyclotron frequency $\omega_{c} / \omega_{p}$, for a fixed shell diameter $b / \lambda_{p}=0.2$ and core-shell ratio $a / b=0.5$. In addition, Fig. 1(b) shows the scattering coefficient magnitude $\left|S_{-1}\right|$ for the same scatterer, varying frequency $\omega / \omega_{p}$ and core-shell ratio $a / b$, for a fixed weak magnetic bias $\omega_{c} / \omega_{p}=0.05$ along the $+z$ axis. The ultralow-frequency resonance predicted by Eq. (3) is visible in the figure as an ultrasharp asymmetric resonant feature standing out against a broad scattering background. Panels (c) and (d) show the total scattering efficiency $\sigma_{2 D} /(2 b)$ for two different values of aspect ratio, revealing the narrow asymmetric line shape of these resonances, similar to the response of sharp Fano resonances and quasiembedded eigenstates in other scattering systems [12-15]. As expected for a scatterer in the lossless limit, the resonance peak value equals the maximum achievable dipolar scattering efficiency for a two-dimensional object, i.e., $\lambda /(\pi b)[16,17]$.

In contrast with any reciprocal plasmonic scatterer, these results theoretically demonstrate that a weakly magnetized plasmonic object with nonsingular geometry can be made to resonate at arbitrarily low frequencies, independently of the plasma frequency of the constituent materials.

\section{A. Relation to topological surface plasmon polaritons}

The physical origin of this "hidden" scattering resonance supported by gyrotropic core-shell structures can be better understood by analyzing the corresponding planar structure, shown in the inset of Fig. 2(c), as usually done to gain physical insight into the resonance mechanism (e.g., [17]). The planar analog consists of a biased plasma half space, covered by a layer of nonbiased plasma of thickness $(b-a)$. This planar configuration belongs to a broad class of stratified gyrotropic-isotropic structures that have been recently the subject of extensive investigations in the context of nonreciprocal and topological electromagnetics [18-30]. Several works have shown that nonreciprocal systems of this type may support unidirectional modes, robust against defects and discontinuities, associated with certain nontrivial topological properties [18-22,31]. Specifically, the bulk modes of a homogeneous material can be characterized by a topological integer number known as Chern number $\mathscr{C}$, which can be intuitively interpreted as a sort of winding number for the modal evolution in momentum space [32-36]. For the structure under consideration, a nonzero Chern number $\mathscr{C} \neq 0$ is associated 

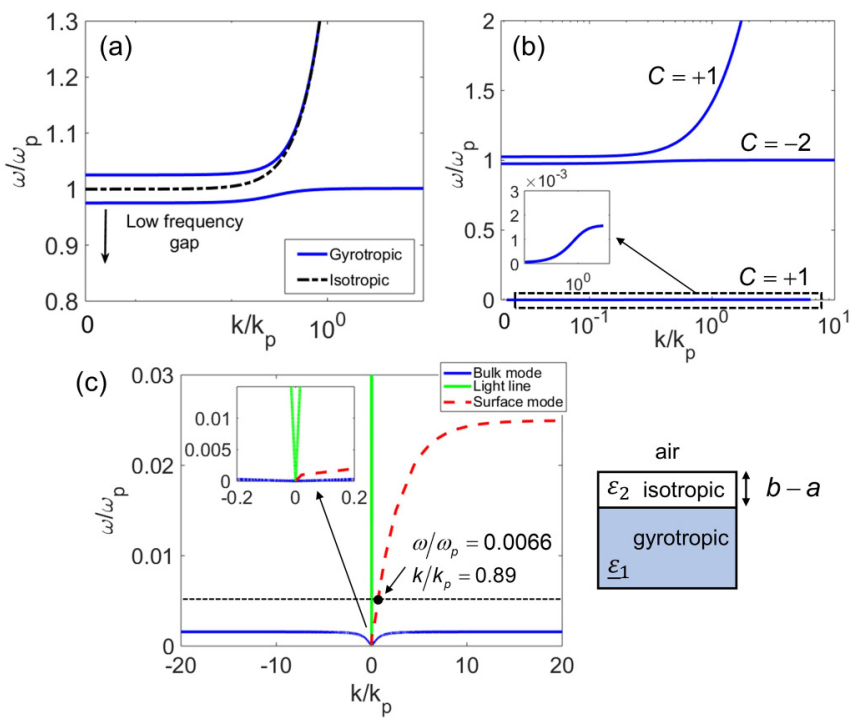

FIG. 2. Modal analysis of a planar analog of the cylindrical scatterer in Fig. 1. (a) Bulk-mode band diagram for a gyrotropic (magnetized) plasmonic material (solid blue) and an isotropic (unmagnetized) plasma (dashed black) with the same plasma frequency $\omega_{p}$, and $\omega_{c} / \omega_{p}=0.05$. (b) Bulk-mode band diagram of the interpolated material model (see explanation in the text), with $\tau=0.001$. The Chern number for each band is indicated. (c) Lowest-frequency bulk band (solid blue) and unidirectional, topologically protected surface mode (dashed red), for the planar structure under consideration (inset to the right) with isotropic-layer thickness $b-a=0.03 \lambda_{p}$. Green lines indicate the light cone in air. All dispersion diagrams are calculated for transverse-magnetic propagation in the plane orthogonal to the bias.

with each bulk band of the gyrotropic half space, as indicated in Fig. 2(b). In contrast, the bulk modes of the isotropic material have $\mathscr{C}=0$, which means that they are topologically trivial. The integer Chern number associated with a bulk band cannot change unless the gap separating it from other bands is closed [18]. As seen in Figs. 2(a) and 2(b), the two considered materials (gyrotropic and isotropic plasmas) share a common bulk-mode band gap at low frequencies, below their plasma frequency. This implies that it is impossible to continuously transform one of the materials into the other, changing its topological properties, without closing the gap. Thus, if the gyrotropic plasmonic material becomes an isotropic plasma, as the magnetic bias drops to zero near the interface, the band gap must close somewhere in the transition region, leading to the emergence of topologically protected surface waves. Such a transition from one medium to the other can be analyzed by considering an interpolated material model with permittivity tensor $\underline{\boldsymbol{\epsilon}}_{\tau}=(1-\tau) \underline{\boldsymbol{\epsilon}}_{1}+\tau \epsilon_{2} \mathbf{I}$. Hence, $\underline{\boldsymbol{\epsilon}}_{\tau}$ represents a continuous transformation between the gyrotropic $(\tau=0)$ and the isotropic plasma $(\tau=1)$.

Figure 2(b) shows the bulk bands of the weakly interpolated model for $\tau=0.001$. As seen in the inset, a nearzero-frequency bulk TM band is present, with Chern number $\mathscr{C}=+1$, as predicted in Refs. [19,22], separated from the higher-frequency bands by a wide band gap. Consistent with our discussion above, a topologically protected surface mode emerges in this band gap, supported by the interface between the two topologically distinct media (biased and nonbiased plasmas), as shown in Fig. 2(c) for a planar structure with the same parameters of the corresponding core-shell cylindrical scatterer. Importantly, we note that this ultralow-frequency surface plasmon-polariton mode is unidirectional. The modal behavior at such low frequencies is crucial to understand and interpret the corresponding scattering resonance of the considered nonreciprocal scatterer. More specifically, if we "bend" the planar geometry in Fig. 2 into a cylindrical shape, the surface wave should reconnect after a full round trip in order to determine a resonance of the cylindrical structure. In other words, the scatterer supports a resonance when the surface-wave wave number $k$ obeys the whispering-gallery mode condition [1],

$$
k(\omega) l=2 \pi n,
$$

where $n$ is the (integer) resonance order and $l=2 \pi a$ is the length (perimeter) of the core-shell interface on which the surface mode is tightly confined [37]. The dispersion relation of the ultralow-frequency surface mode in Fig. 2 together with the whispering-gallery mode condition in Eq. (5) allow predicting the scattering resonance observed in Fig. 1. For example, at $\omega / \omega_{p} \approx 0.006625$, where the scattering resonance of Fig. 1(c) occurs, the surface mode dispersion in Fig. 2(c) gives $k / k_{p} \approx 0.89$, where $k_{p}=2 \pi / \lambda_{p}$. According to (5), this mode produces a resonance of order $n=1$ only if $a \approx 0.178 \lambda_{p}$, which agrees well with the radius of the considered coreshell structure in Fig. 1(c). This confirms our interpretation that the "hidden" ultralow-frequency resonance considered in this work originates from a unidirectional and topologically protected surface plasmon polariton, which exists, at very low frequencies, on a partially magnetized plasmonic object. We also note that this surface mode has been shown in several papers (e.g., [18,28]) to exist if a magnetized plasma is interfaced with a generic opaque material, that is, a perfect or imperfect conductor, or a plasmonic material below its plasma frequency. Thus, even if the plasma frequency of the shell material was changed, the unidirectional surface plasmonpolariton mode would still exist, with a slightly different dispersion, which would result in a scattering resonance with slightly different frequency, but otherwise mostly unaltered.

Figure 3(a) shows the total magnetic field distribution and the Poynting vector streamlines around the magnetized plasma cylinder in Fig. 1, illuminated by an incident plane wave with unit amplitude, at the resonance frequency identified in Fig. 1(c). Despite the deeply subwavelength size of the scatterer $(b / \lambda \approx 0.0013)$ and the fact that we are operating at a frequency more than a hundred times smaller than $\omega_{p}$, the scatterer produces a large disturbance in the spatial profile of the field and power flow, a clear signature of the presence of a strong resonance. Figure 3(b) shows the scattered field, and the inset provides a zoomed-in view of the fields near and inside the scatterer, revealing the dipolar nature of the resonance (corresponding to $S_{-1}$ ). The observed vortexlike scattering, different from the conventional pattern of dipolar scattered fields, is due to the absence of a frequencydegenerate resonance associated with the opposite dipolar term $S_{+1}$. Specifically, when only one term $\left(S_{ \pm 1}\right)$ is present, the response is dominated by a circularly polarized induced dipole of certain handedness or, equivalently, two orthogonal 

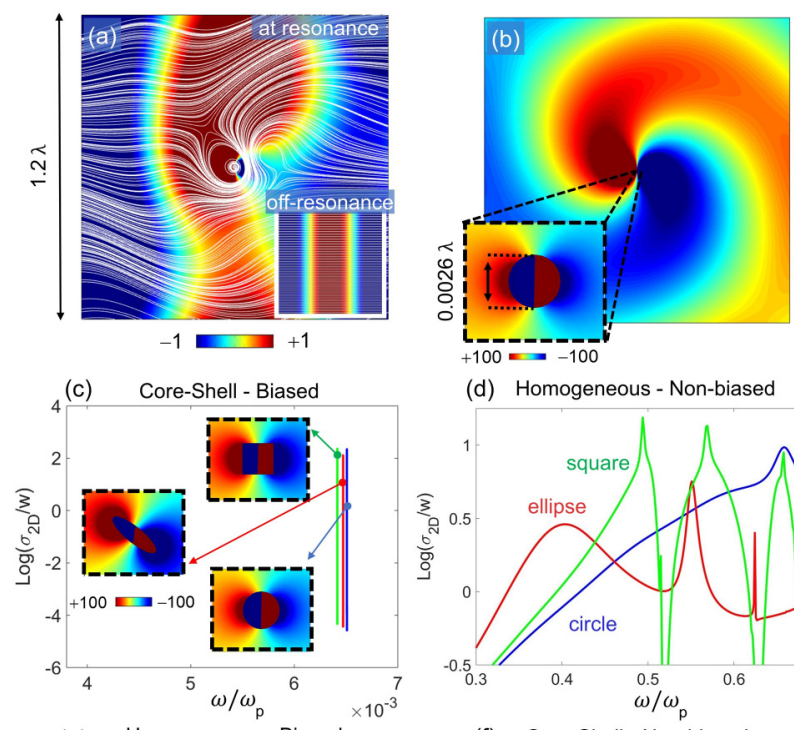

(d) Homogeneous - Non-biased

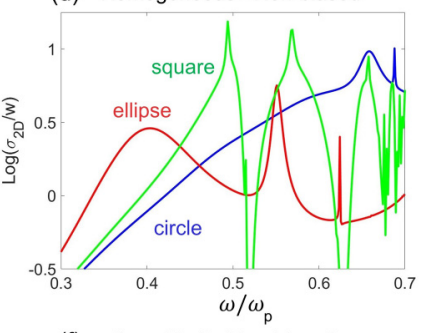

(e) Homogeneous - Biased

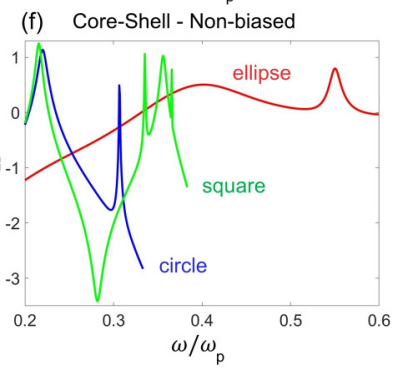

FIG. 3. (a) Total magnetic field distribution (time snapshot of the out-of-plane field component) and Poynting vector streamlines, for the cylindrical core-shell structure of Fig. 1(c), at resonance. For comparison, the inset shows the off-resonance case for the same object. (b) Similar to (a), but for the scattered field. The inset shows a zoomed-in view of the scatterer and its dipolar field distribution. (c) Resonance peaks of the scattering efficiency for scatterers with circular [as in Fig. 1(c)], elliptical, and square cross sections, all having the same perimeter $l$. Insets show the scattered field distribution near the scatterers. (d), (e), (f) Similar to panel (c), but for different configurations of interest: (d) nonmagnetized homogeneous plasmonic scatterer; (e) magnetized homogenous plasmonic scatterer; (f) nonmagnetized core-shell scatterer with dielectric core and thin plasmonic shell. For all these cases, the outer radius of the scatterer is the same, and we used the same plasmonic material with the same plasma frequency. For panels (c), (e), the magnetic bias is the same. For panels (c), (f), the thickness of the shell is the same.

linear dipoles oscillating in quadrature $( \pm \pi / 2)$. This behavior leads to a vortexlike scattered field carrying a nonzero angular momentum, either +1 or -1 , in contrast with the degenerate angular momentum channels of isotropic scatterers with cylindrical symmetry.

\section{B. Shape-independent scattering response}

Another interesting feature of such ultralow-frequency scattering resonances, stemming from the topological nature of the low-frequency surface mode, is their robustness against perturbations of the shape of the core-shell resonator. In fact, the geometrical cross section of the scatterer can be modified to a large extent, while robustly preserving the same resonance frequency and line shape, as long as the perimeter of the scatterer is kept fixed, similar to recently studied shape-independent topological cavities [38,39]. Thanks to its topological properties, the surface mode does not backscatter at imperfections, discontinuities, and sharp bends, which may otherwise disrupt the resonance properties of the scatterer as multiple reflections would determine different optical paths seen by the surface wave, giving rise to different resonances.

To demonstrate the robustness of the scattering response, we deformed the circular cross section of the considered cylindrical scatterer into an elongated ellipse and a square, while retaining the same perimeter and, therefore, the same path length $l=2 \pi a$ seen by the surface mode. Figure 3(c) shows the resonant peaks of the scattering efficiency $\sigma / w(w$ stands for the width of each object in the direction of the incident wave) for these three scatterers of different shape. As clearly seen in this figure, drastically reshaping the object only slightly alters the resonance frequency. The ultranarrow line shape and peak value $\lambda /(\pi b)$ of these dipolar resonances are also preserved. This behavior originates from the highly confined unidirectional surface mode internally supported by our core-shell structure, which makes the resonance dependent only on the perimeter, not the shape of the scatterer. In contrast, dipolar scattering resonances supported by more conventional reciprocal or nonreciprocal plasmonic scatterers are strongly affected by the shape of the particle. Figures 3(d)3(f) show different cases of interest: (d) Homogeneous, nonmagnetized, plasmonic cylinders with the same plasma frequency and dimensions as in panel (c). The dipolar scattering resonance (broader peak) is close to the plasma frequency, consistent with Eq. (1), and is strongly affected by a change of geometrical cross section, both in terms of resonance frequency and line shape. Since the object is not deeply subwavelength at frequencies near $\omega_{p}$, additional sharper peaks are visible, due to multipolar resonances. (e) Homogeneous, magnetized, plasmonic cylinders with the same plasma frequency, cyclotron frequency, and dimensions as in panel (c). Consistent with Eq. (2) for a circular cylinder, the dipolar peak splits in two due to the magnetization (Zeeman effect). As in panel (d), the resonances depend strongly on the shape of the scatterer. (f) Nonmagnetized core-shell scatterer with a dielectric core $(\epsilon=2)$, and a plasmonic shell having the same thickness and plasma frequency as in panel (c). Consistent with Eq. (4) and our discussion in the Introduction, a thin plasmonic shell does shift the resonance at lower frequencies, but not nearly as much as in the case of the magnetized core-shell scatterer in panel (c) for the same thickness. While in this scenario the modification from circular to square cross section perturbs the resonance only slightly, a change from circular to elliptical cross section leads to a large perturbation of the resonance frequency and line shape.

These results clearly show that only the proposed gyrotropic configuration in panel (c) is very robust to generic perturbations of the shape of the scatterer.

\section{Superscattering effects}

The scattering effects discussed above are not limited to dipolar resonances. Indeed, the flattening of the surface-mode dispersion in Fig. 2(c) for large values of $k / k_{p}$ suggests the possibility that the whispering-gallery mode condition in 


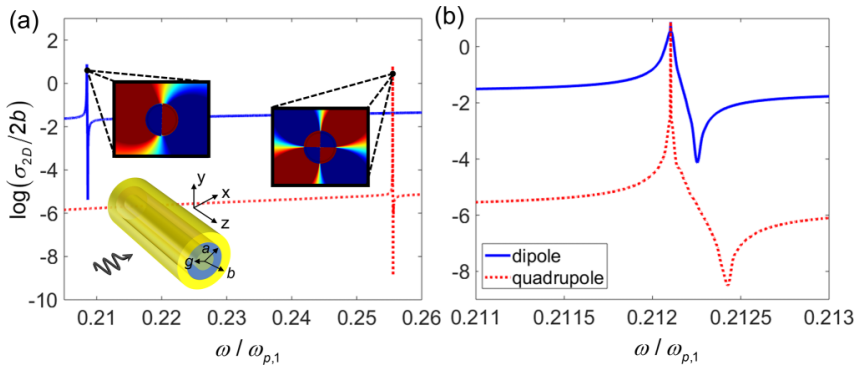

FIG. 4. Superscattering effects. (a) Dipole (solid blue) and quadrupole (dotted red) scattering efficiency for the three-layer structure shown in the inset (details in the text). Parameters: $\omega_{p, 2} / \omega_{p, 1}=$ $11, \omega_{p, 3} / \omega_{p, 1}=2.72, \omega_{c, 1} / \omega_{p, 1}=\omega_{c, 3} / \omega_{p, 1}=0.3, \quad b / \lambda_{p, 1}=0.2$, $a / b=0.9$, and $g / b=0.85$, where $\omega_{p, j}, j=1,2,3$ are the plasma frequencies of the core, intermediate layer, and outer shell, $\omega_{c, j}, j=$ 1,3 are the cyclotron frequencies of the core and outer shell, and $g, a, b$ are the radii of the core, intermediate layer, and outer shell, respectively. $\lambda_{p, 1}$ is the free-space wavelength at $\omega_{p, 1}$. Insets show the scattered field distribution near the layered cylinder. (b) Similar to panel (a), but for a superscattering configuration with dipole and quadrupole resonances at the same frequency. The structure parameters are the same as in (a), except $\omega_{p, 2} / \omega_{p, 1}=12$.

Eq. (5) may be satisfied for different values of $n>1$ over a narrow frequency window, leading to the spectral overlap of multiple scattering resonances of different order and angular momentum. This is indeed the mechanism behind socalled superscatterers, first demonstrated in Ref. [17], which allow overcoming the single-channel limit of the scattering efficiency $[\lambda /(\pi b)$ for a two-dimensional (2D) object of width $2 b$ ] by resonantly exciting different angular momentum channels at the same frequency. However, we found that our simple core-shell structure studied above does not offer enough degrees of freedom to control and induce such a resonance superposition. Inspired by the geometry studied in Ref. [17], we therefore considered the three-layer structure illustrated in the inset of Fig. 4(a), where the inner core and outer shell are gyrotropic while the intermediate layer is isotropic. This extra layer enables the emergence of two coupled surface modes supported by the two inner cylindrical interfaces, allowing control of their dispersion with more flexibility. Figure 4(a) shows an example of the resulting scattering efficiency spectrum for two different angular momentum channels, revealing the presence of both a dipole and a quadrupole resonance, at two separate but nearby frequencies (the insets show the corresponding field profiles). By suitably engineering and optimizing the geometry and material properties of the layers, it is then possible to bring these two resonances at the same frequency, as demonstrated in Fig. 4(b), producing superscattering effects. It should be stressed that, while the design considered in Fig. 4(b) achieves resonance superposition at a frequency only moderately smaller than the plasma frequency $\left(\omega \approx \omega_{p} / 5\right)$, it is in principle possible to push this superscattering effect to arbitrarily low frequencies, similar to the dipolar scattering results in Fig. 1. We expect that the topological properties of these resonances may be beneficial to the realization of robust superscattering effects, which are typically very sensitive to perturbations and defects that detune the resonances.

\section{CONCLUSION}

In this paper, we have investigated the physical mechanism at the origin of a class of anomalous ultralow-frequency plasmonic resonances, shedding light onto their relation to unidirectional and topological surface plasmon polaritons supported by nonreciprocal plasmonic platforms. These topological properties endow the scattering resonance with robustness against perturbations and geometrical modifications. While we expect that the effect of material absorption may be important in solid-state plasmonic media at room temperature, we predict that these scattering effects could be observed in low-loss plasmonic (meta)materials and gas plasmas under weak magnetization, including in atmospheric and astrophysical settings where rarefied plasmas under magnetic bias are a common occurrence. Further details on the practical feasibility of the proposed scatterers and on the impact of dissipation are provided in Appendix C.

In summary, our findings describe a class of anomalous low-frequency scattering resonances and show an intriguing connection between topological photonics/electromagnetics and scattering problems.

\section{ACKNOWLEDGMENTS}

S.A.H.G. and F.M. acknowledge support from the National Science Foundation (NSF) with Grant No. 1741694, and the Air Force Office of Scientific Research with Grant No. FA9550-19-1-0043.

\section{APPENDIX A: DIPOLAR SCATTERING COEFFICIENTS FOR A CORE-SHELL GYROTROPIC SCATTERER}

Consider a generic, infinitely long, circular cylinder in free space with axis along the $z$ direction (see Fig. 1). When a transverse-magnetic (TM) plane wave with magnetic field along the cylinder axis illuminates the scatterer, the scattered field into free space can be represented as a discrete sum of cylindrical harmonics, $H_{z}^{s}=\sum_{m=-\infty}^{+\infty} S_{m} H_{m}^{(2)}\left(k_{0} r\right) e^{i m \phi}$, where $\phi$ is the cylindrical coordinate angle measured from the $+x$ axis, $H_{m}^{(2)}$ is the $m$ th order Hankel function of the second kind representing outgoing waves, and $S_{m}$ is referred to as the TM scattering coefficient. Writing the incident plane wave in the form $H_{z}=H_{0} e^{-i \mathbf{k}_{0} . \mathbf{r}}$, with $H_{0}$ being the magnetic field amplitude, the scattering width takes the form $\sigma_{2 D}=$ $\frac{2 \lambda}{\pi} \sum_{m=-\infty}^{+\infty}\left|S_{m}\right|^{2}$. By normalizing $\sigma_{2 D}$ to the width of the object, $2 b$, it can be seen that the theoretical upper bound of the scattering efficiency for any $m$ th angular momentum channel is $\lambda / \pi b$ for $2 \mathrm{D}$ objects.

Since we are interested in small, subwavelength scatterers, we make a quasistatic approximation to simplify the expressions of the scattering coefficients. Mathematically, the quasistatic analysis is based on a small-argument approximation for the Bessel and Hankel functions in a Mie-theory formulation of the scattering coefficients (corresponding, physically, to neglecting retardation effects). The Bessel/Hankel function arguments entering the formulation are, in free space, $k_{0} b=2 \pi b / \lambda_{0}$ and, in the magnetized plasma, $k_{0} b \sqrt{\epsilon_{\mathrm{eff}}}=$ $k_{0} b \sqrt{\left(\epsilon_{t}^{2}-\epsilon_{g}^{2}\right) / \epsilon_{t}}$, where $\epsilon_{t}$ and $\epsilon_{g}$ are the diagonal and 
off-diagonal permittivity tensor elements. In the limit of small frequency and small cyclotron frequency (with respect to the plasma frequency), it is straightforward to show that $k_{0} b \sqrt{\epsilon_{\mathrm{eff}}}=2 \pi i b / \lambda_{p}$, where $\lambda_{p}$ is the free-space wavelength at the plasma frequency. Thus, if the radius $b$ is smaller than both the free-space wavelength at the operational frequency and the free-space wavelength at the plasma frequency, a small-argument, quasistatic approximation of the scattering coefficients is valid. This is indeed the case in our work, which explains why the quasistatic formulas agree well with full-wave calculations (Fig. 1) and numerical simulations (Fig. 3).

As explained in the main text, in the quasistatic limit the dominant scattering coefficients are the $S_{ \pm 1}$ ones, corresponding to dipolar scattered waves of equal and opposite angular momentum. Applying Mie theory to the core-shell gyrotropic cylinder considered in the main text, in the long-wavelength regime, we find the following approximate expression for the dipolar scattering coefficients (further details are available in Ref. [10]):

$$
S_{ \pm 1} \approx \pi^{3}\left(\frac{b}{\lambda_{p}}\right)^{2}\left(\frac{\omega}{\omega_{p}}\right)^{2} \times \frac{\frac{\omega_{c}}{\omega_{p}}\left[\left(\frac{a}{b}\right)^{2}-1\right]\left[1-2\left(\frac{\omega}{\omega_{p}}\right)^{2}\right] \pm 2 \frac{\omega}{\omega_{p}}\left[\left(\frac{\omega}{\omega_{p}}\right)^{2}-1\right]}{2 \frac{\omega}{\omega_{p}}\left[1-\left(\frac{\omega}{\omega_{p}}\right)^{2}\right]\left[1-2\left(\frac{\omega}{\omega_{p}}\right)^{2}\right] \mp \frac{\omega_{c}}{\omega_{p}}\left\{\left(\frac{a}{b}\right)^{2}-\left[1-\left(\frac{\omega}{\omega_{p}}\right)^{2}\right]^{2}\right\}},
$$

where $\omega_{c}$ is the cyclotron frequency (bias applied only to the core region) and $\omega_{p}$ is the common plasma frequency of the entire plasmonic cylinder. In the above equation, the roots of the denominator indicate the frequencies at which scattering resonances occur (in the quasistatic regime, neglecting radiation loss, scattering resonances and poles coincide). Due to the presence of the bias, $\omega_{c} \neq 0$, the degeneracy of dipolar resonances with opposite angular momentum is lifted. This situation is similar to the case of a biased homogeneous plasmonic cylinder; however, as discussed in the main text, a partially magnetized plasmonic scatterer supports additional ultralow-frequency resonances associated with ultralow-frequency topological surface modes supported by the internal interface.

\section{APPENDIX B: POTENTIAL IMPLEMENTATION OF THE PROPOSED SCATTERER}

The biased portion of the proposed scatterer could be realized in the form of $n$-doped semiconductors, e.g., $n$-type InSb, which is a popular model system for nonreciprocal plasmonics since the 1970s $[25,28,40,41]$. The plasma frequency of InSb with electron density of $10^{22}$ per cubic meters is equal to $\omega_{p} / 2 \pi=2 \mathrm{THz}$. Considering the low effective electron mass of $\operatorname{InSb}\left(m^{*} / m=0.0142\right)$, we find that it is possible to obtain sufficiently large cyclotron frequency with low values of bias. Specifically, the strength of the magnetic field in our Figs. 1 to 3 of the main text is about $0.05 \mathrm{~T}$, and for the results of Fig. 4 it is about $0.3 \mathrm{~T}$. These levels of bias intensity can be achieved even with commercially available permanent magnets or simple electromagnets.

Regarding the implementation of the bias, there are at least two strategies that could be used to practically implement a cylindrical configuration with biased core and nonbiased shell. For example, by loosely wrapping a solenoid around the core region (cylinder of radius $a$ ), with DC current $I$, it would be possible to create a magnetic field that is almost completely confined within the core region, as long as its length $L$ is much larger than $a$. In addition, if the windings are sufficiently separated and/or tilted, their effect on the incident harmonic wave may be reduced. If one denotes by $N$ the solenoid loops per unit length along the $z$ axis, the DC magnetic field inside the long solenoid $(r<a)$ is almost homogeneous, especially if our observation plane, e.g., $z=0$, is far from the cylinder ends. The value of the internal field is given by $B_{0}=N I \mu_{0}$, and the field is parallel to the $z$ axis. However, due to its finite size, the magnetic field outside the solenoid $(r>a)$ is non-zero since the magnetic field lines must form closed loops. The axial magnetic field $B_{z}$ in the region $r>a$ on the plane $z=0$ can be determined as follows $[42,43]$ :

$$
\frac{B_{z}}{B_{0}}=\frac{2(a / L)^{2}}{\sqrt{\left[1+4(r / L)^{2}\right]^{3}}},
$$

where $r$ is the radial distance. It is clear that, if $a \ll L$, the external field, $B_{z}$, is much smaller than the homogeneous field $B_{0}$ inside the core; therefore, its effect on the properties of the shell material will be quite limited and may be ignored. In other words, the gyrotropic character of the shell region $(a<r<b)$ will be weak since the magnetic lines form very sparsely distributed closed loops across a semi-infinite region, yielding a particularly low magnetic field.

We also would like to stress that, in the main text, we have considered a structure with the same plasma frequency for the core and shell in order to simplify the analysis and gain more physical insight from the resulting simple analytical expressions. However, having exactly the same plasma frequency for the core and shell is not a requirement to obtain the observed ultralow-frequency scattering resonances. These resonances, like all plasmonic resonances, originate from surface plasmon polaritons existing on the interfaces of the scatterer. As mentioned in the main text, the unidirectional and topological mode that is responsible for these scattering features has been shown in several papers to exist if a magnetized plasma is interfaced with a generic opaque material. Indeed, the topological nature of these modes is retained as long as the two materials at the interface have overlapping bulk-mode band gaps [19]. Thus, even if the plasma frequency of the shell was different, the unidirectional surface plasmon-polariton mode would still exist, with a slightly different dispersion, resulting in a scattering resonance with slightly different frequency, but otherwise mostly unaltered. Indeed, an even simpler strategy to practically realize the proposed configuration is to consider a core-shell cylinder that is uniformly magnetized, but with the shell composed of a different material with much higher plasma frequency and/or much larger electron effective mass. 
In this way, for the same level of magnetic bias, the cyclotron frequency normalized by the plasma frequency, $\omega_{c} / \omega_{p}$, in the shell would be much lower than in the core, and could be neglected. In other words, despite the presence of the bias, the permittivity of the shell would be essentially scalar, with negligible nonreciprocal effects.

\section{APPENDIX C: IMPACT OF DISSIPATION}

The ultrasharp scattering resonances described in the main text are very sensitive to absorption loss. However, we would like to note that, in this work, we have considered particularly extreme cases. Indeed, these topological resonances do not have to be at such low frequencies. They can occur at frequencies closer to the plasma frequency where the scattering peak becomes wider and less sensitive to the effect of losses. In Fig. 5, we show an example of the impact of absorption losses on the scattering resonance, calculated exactly using Mie theory. We consider InSb with plasma frequency as in Appendix B and cyclotron frequency $\omega_{c}=0.5 \omega_{p}$. The considered losses correspond to collision frequencies $2 \gamma=$ $10^{-6} \omega_{p}, 10^{-5} \omega_{p}, 10^{-4} \omega_{p}$, which are potentially feasible for $\mathrm{InSb}$ at liquid-nitrogen temperatures $[44,45]$ or for gas plasmas. The permittivity tensor elements in the presence of loss take the form

$$
\epsilon_{t, 1}=1-\frac{\omega_{p}^{2}\left(1-i \frac{\gamma}{\omega}\right)}{(\omega-i \gamma)^{2}-\omega_{c}^{2}}, \quad \epsilon_{g, 1}=\frac{\omega_{p}^{2} \omega_{c}}{\omega\left[\omega_{c}^{2}-(\omega-i \gamma)^{2}\right]} .
$$

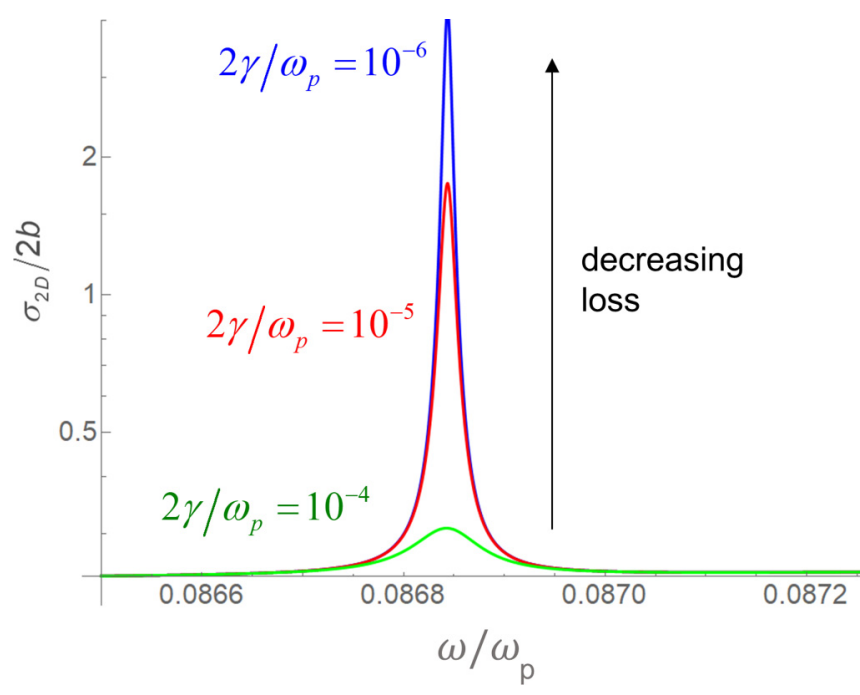

FIG. 5. Impact of dissipation on the low-frequency scattering resonance of the proposed gyrotropic core-shell particle, for three different levels of absorption loss.

From these results, it is clear that the scattering resonance remains fairly strong for a reasonably wide range of Ohmic losses. Low-loss plasmas are available in nature in the form of low-temperature solid-state plasmas, low-loss plasmonic metamaterials (e.g., wire media), and rarefied gas plasmas.
[1] L. Novotny and B. Hecht, Principles of Nano-Optics (Cambridge University Press, Cambridge, 2006), Chap. 12.

[2] S. A. Maier, Plasmonics: Fundamentals and Applications (Springer, Berlin, 2007), Chap. 5.

[3] A. Sihvola, in Electromagnetic Mixing Formulas and Applications (IEE, London, 1999), Chap. 4, pp. 63-67.

[4] D. Tzarouchis and A. Sihvola, General scattering characteristics of resonant core-shell spheres, IEEE Trans. Antennas Propag. 66, 323 (2018).

[5] A. Ishimaru, in Electromagnetic Wave Propagation, Radiation, and Scattering (Prentice Hall, Upper Saddle River, NJ, 1991), Chap. 8, pp. 220-223.

[6] C. F. Bohren and D. R. Huffman, Absorption and Scattering of Light by Small Particles (John Wiley \& Sons, Hoboken, 2008).

[7] J. B. Pendry, A. J. Holden, W. J. Stewart, and I. Youngs, Extremely Low Frequency Plasmons in Metallic Meso-Structures, Phys. Rev. Lett. 76, 4773 (1996).

[8] R. A. Shelby, D. R. Smith, and S. Schultz, Experimental verification of a negative index of refraction, Science 292, 77 (2001).

[9] J. Monzon and N. Damaskos, Two-dimensional scattering by a homogeneous anisotropic rod, IEEE Trans. Antennas Propag. 34, 1243 (1986).

[10] C. Valagiannopoulos, S. A. H. Gangaraj, and F. Monticone, Zeeman gyrotropic scatterers: Resonance splitting, anomalous scattering, and embedded eigenstates, Nanomater. Nanotechnol. 8, 1 (2018).
[11] F. Monticone and A. Alu, Scattering at the extreme with metamaterials and plasmonics, in Handbook of Metamaterials and Plasmonics, edited by S. Maier (World Scientific, Singapore, 2017), Vol. 1, Chap. 7, pp. 295-335.

[12] J. B. Lassiter, H. Sobhani, J. A. Fan, J. Kundu, F. Capasso, P. Nordlander, and N. J. Halas, Fano resonances in plasmonic nanoclusters: Geometrical and chemical tunability, Nano Lett. 10, 3184 (2010).

[13] F. Monticone and A. Alu, Embedded Photonic Eigenvalues in 3D Nanostructures, Phys. Rev. Lett. 112, 213903 (2014).

[14] A. E. Miroshnichenko, S. Flach, and Y. S. Kivshar, Fano resonances in nanoscale structures, Rev. Mod. Phys. 82, 2257 (2010).

[15] B. Luk'yanchuk, N. I. Zheludev, S. A. Maier, N. J. Halas, P. Nordlander, H. Giessen, and C. T. Chong, The Fano resonance in plasmonic nanostructures and metamaterials, Nat. Mater. 9, 707 (2010).

[16] R. E. Hamam, A. Karalis, J. D. Joannopoulos, and M. Soljacic, Coupled-mode theory for general free-space resonant scattering of waves, Phys. Rev. A 75, 053801 (2007).

[17] Z. Ruan and S. Fan, Superscattering of Light from Subwavelength Nanostructures, Phys. Rev. Lett. 105, 013901 (2010).

[18] M. G. Silveirinha, Chern invariants for continuous media, Phys. Rev. A 92, 125153 (2015).

[19] M. G. Silveirinha, Bulk edge correspondence for topological photonic continua, Phys. Rev. B 94, 205105 (2016). 
[20] M. G. Silveirinha, Proof of the Bulk-Edge Correspondence through a Link between Topological Photonics and FluctuationElectrodynamics, Phys. Rev. X 9, 011037 (2019).

[21] S. A. H. Gangaraj, M. G. Silveirinha, and G. W. Hanson, Berry phase, Berry connection, and Chern number for a continuum bianisotropic material from a classical electromagnetics perspective, IEEE J. Multiscale Multiphys. Comput. Technol. 2, 3 (2017).

[22] S. A. H. Gangaraj, A. Nemilentsau, and G. W. Hanson, The effects of three-dimensional defects on one-way surface plasmon propagation for photonic topological insulators comprised of continuum media, Sci. Rep. 6, 30055 (2016).

[23] K. Tsakmakidis, L. Shen, S. Schulz, X. X. Zheng, J. Upham, X. Deng, H. Altug, A. Vakakis, and R. Boyd, Breaking Lorentz reciprocity to overcome the time-bandwidth limit in physics and engineering, Science 356, 1260 (2017).

[24] S. A. Mann, D. L. Sounas, and A. Alu, Nonreciprocal cavities and the time-bandwidth limit, Optica 6, 104 (2019).

[25] S. Buddhiraju, Y. Shi, A. Song, C. Wojcik, M. Minkov, I. A. D. Williamson, A. Dutt, and S. Fan, Absence of unidirectionally propagating surface plasmon-polaritons at nonreciprocal metal-dielectric interfaces, Nat. Commun. 11, 674 (2020).

[26] A. Davoyan and N. Engheta, Theory of Wave Propagation in Magnetized Near-Zero-Epsilon Metamaterials: Evidence for One-Way Photonic States and Magnetically Switched Transparency and Opacity, Phys. Rev. Lett. 111, 257401 (2013).

[27] J. Khurgin, Optical isolating action in surface plasmon polaritons, Appl. Phys. Lett. 89, 251115 (2006).

[28] S. A. H. Gangaraj and F. Monticone, Do truly unidirectional surface plasmon-polaritons exist? Optica 6, 1158 (2019).

[29] S. A. H. Gangaraj and F. Monticone, Physical Violations of the Bulk-Edge Correspondence in Topological Electromagnetics, Phys. Rev. Lett. 124, 153901 (2020).

[30] T. V. Mechelen and J. Zubin, Unidirectional Maxwellian spin waves, Nanophotonics 8, 1399 (2019).

[31] S. A. H. Gangaraj and F. Monticone, Topologically-protected one-way leaky waves in nonreciprocal plasmonic structures, J. Phys.: Condens. Matter 30, 104002 (2018).

[32] Z. Wang, Y. D. Chong, J. D. Joannopoulos, and M. Soljacic, Reflection-Free One-Way Edge Modes in a Gyromagnetic Photonic Crystal, Phys. Rev. Lett. 100, 013905 (2008).

[33] Z. Yu, G. Veronis, Z. Wang, and S. Fan, One-Way Electromagnetic Waveguide Formed at the Interface between a Plasmonic
Metal under a Static Magnetic Field and a Photonic Crystal, Phys. Rev. Lett. 100, 023902 (2008).

[34] Z. Wang, Y. Chong, J. D. Joannopoulos, and M. Soljacic, Observation of unidirectional backscattering-immune topological electromagnetic states, Nature (London) 461, 772 (2009).

[35] M. C. Rechtsman, J. M. Zeuner, Y. Plotnik, Y. Lumer, D. Podolsky, F. Dreisow, S. Nolte, M. Segev, and A. Szameit, Photonic Floquet topological insulators, Nature (London) 496, 196 (2013)

[36] M. C. Rechtsman, Y. Plotnik, J. M. Zeuner, D. Song, Z. Chen, A. Szameit, and M. Segev, Topological Creation and Destruction of Edge States in Photonic Graphene, Phys. Rev. Lett. 111, 103901 (2013).

[37] We would like to note that the perimeter is proportional to the optical path only if the surface wave is well confined on the cylindrical interface and does not couple with the surface wave propagating on the other side of the scatterer (we have verified that this is true in our case). The system is therefore robust to geometrical perturbations only as long as the scatterer does not include narrow segments where counterpropagating unidirectional surface waves can couple through the core of the object, which may lead to a moderate modification of the dispersion diagram of these coupled surface modes.

[38] B. Bahari, A. Ndao, F. Vallini, A. El Amili, Y. Fainman, and B. Kante, Nonreciprocal lasing in topological cavities of arbitrary geometries, Science 358, 636 (2017).

[39] M. A. Bandres, S. Wittek, G. Harari, M. Parto, J. Ren, M. Segev, D. N. Christodoulides, and M. Khajavikhan, Topological insulator laser: Experiments, Science 359, eaar4005 (2018).

[40] J. J. Brion, R. F. Wallis, A. Hartstein, and E. Burstein, Theory of Surface Magneto Plasmons in Semiconductors, Phys. Rev. Lett. 28, 1455 (1972).

[41] A. Hartstein and E. Burstein, Observation of magnetoplasma type surface polaritons on $n$-InSb, Solid State Commun. 14, 1223 (1974).

[42] E. E. Callaghan and S. H. Maslen, The magnetic field of a finite solenoid, NASA Technical Note, 1960, https://ntrs.nasa. gov/search.jsp?R=19980227402

[43] A. Pathak, An elementary argument for the magnetic field outside a solenoid, Eur. J. Phys. 38, 015201 (2017).

[44] J. J. Whalen and C. R. Westgate, Temperature dependence of the energy relaxation time in $n$-InSb, Appl. Phys. Lett. 15, 292 (1969).

[45] D. N. J. Cheeke, G. Madore, and A. Hikata. Magnetic field dependent relaxation time in $\mathrm{p}$-InSb at low temperatures, J. Phys. Colloq. 42, C5-689 (1981). 